\title{
Expression of Two Functionally Distinct Plant Endo- $\beta-1,4-$ Glucanases Is Essential for the Compatible Interaction Between Potato Cyst Nematode and Its Hosts
}

\author{
Aneta Karczmarek, ${ }^{1,2}$ Sylwia Fudali, ${ }^{3}$ Malgorzata Lichocka, ${ }^{3}$ Miroslaw Sobczak, ${ }^{3}$ Wojciech Kurek, ${ }^{3}$ \\ Slawomir Janakowski, ${ }^{3}$ Jan Roosien, ${ }^{1}$ Wladyslaw Golinowski, ${ }^{3}$ Jaap Bakker, ${ }^{1}$ Aska Goverse, ${ }^{1}$ and \\ Johannes Helder ${ }^{1}$ \\ ${ }^{1}$ Laboratory of Nematology, Department of Plant Sciences, Wageningen University, Binnenhaven 5, 6709 PD Wageningen, \\ The Netherlands; ${ }^{2}$ Molecular Cytology, Swammerdam Institute for Life Sciences, Faculty of Science, University \\ of Amsterdam, Kruislaan 316, 1098 SM Amsterdam, The Netherlands; ${ }^{3}$ Department of Botany, Faculty of Agriculture \\ and Biology, Warsaw University of Life Sciences (SGGW), Nowoursynowska 159, Building 37, 02-776 Warsaw, Poland
}

Submitted 20 July 2007. Accepted 15 February 2008.

For the proliferation of their feeding sites (syncytia), the potato cyst nematode Globodera rostochiensis is thought to recruit plant endo- $\beta$-1,4-glucanases (EGases, EC. 3.2.1.4). Reverse-transcription polymerase chain reaction experiments on tomato (Solanum lycopersicum) indicated that the expression of two out of the at least eight EGases, namely Sl-cel7 and Sl-cel9C1, is specifically upregulated during syncytium formation. In situ hybridization and immunodetection studies demonstrated that both EGases are specifically expressed inside and adjacent to proliferating syncytia. To assess the importance of $\mathrm{Sl}$-cel7 and $\mathrm{Sl}$-cel9C1 for nematode development, we decided to knock them out individually. Sl-cel9C1 probably is the only class C EGase in tomato, and we were unable to regenerate $\mathrm{Sl}$-cel9C1silenced plants. Potato ( $S$. tuberosum), a close relative of tomato, harbors at least two class $\mathrm{C}$ EGases, and St-cel7or St-cel9C1-silenced potato plants showed no obvious aberrant phenotype. Infection with potato cyst nematodes resulted in a severe reduction of the number of adult females (up to $60 \%$ ) and a sharp increase in the fraction of females without eggs (up to $89 \%$ ). Hence, the recruitment of CEL7, an enzyme that uses xyloglucan and noncrystalline cellulose as natural substrates, and CEL9C1, an enzyme that uses crystalline cellulose, is essential for growth and development of potato cyst nematodes.

Additional keywords: cellulase, cell wall, plant-parasitic nematode, plant resistance.

Cyst nematodes are obligate and usually oligophagous parasites of a number of major crops: the soybean cyst nematode Heterodera glycines is one of the main yield-limiting factors in soybean (Glycine max), potato production worldwide is limited by the potato cyst nematodes Globodera rostochiensis and G. pallida, and sugar beet (Beta vulgaris) is parasitized extensively by $H$. schachtii. As a common denominator, the main

Corresponding author: J. Helder; E-mail: Hans.Helder@wur.nl

* The $e$-Xtra logo stands for "electronic extra" and indicates that one supplemental figure and two supplemental tables are published online. production areas of these crops are usually at a large distance from the areas where co-evolution between the host plants and their parasites took place. The lack of natural enemies and the shortage of resistance genes in crop plants constitute the main factors that underlay the very substantial damage caused by these organisms. Fundamental understanding of the molecular maneuvering of host plants by cyst nematodes could be a starting point for novel kinds of host plant resistances. Here, we focus on plant cell wall manipulation by cyst nematodes.

Cyst nematodes affect host plant cell walls at two distinct points during the infection process. First, cell walls constitute the main physical barrier that should, at least partially, be disassembled during nematode invasion and penetration. Subsequently, the development of cyst nematode feeding sites, actually the fusion of plant protoplasts, is accompanied by local breakdown and remodeling of cell walls. The primary cell wall is a dynamic structure composed of crystalline cellulose microfibrils embedded in a matrix of polysaccharides: hemicelluloses and pectins containing smaller quantities of structural proteins (Carpita and Gibeaut 1993). Plant-parasitic nematodes are equipped with a protrusible stylet (comparable to a hollow needle) that is used during host invasion to puncture the cell wall in a highly coordinated manner. In addition, nematodes also secrete a set of cell-wall-degrading and modifying proteins such as endo- $\beta$-1,4-glucanases (EGases) (Goellner et al. 2000; Smant et al. 1998), pectate lyases (Kudla et al. 2007; Popeijus et al. 2000), and expansins (Qin et al. 2004). Once an infective second-stage juvenile (J2) has entered the plant root, it selects an initial feeding cell (IFC). Highly directed cell wall dissolution between the IFC and adjacent cells results in the formation of a syncytium that is, for the better part, situated within the stele. Finally, the multinucleate syncytium may include up to 200 plant cells (Jones 1981). It is composed of redifferentiated, hypertrophied, and metabolically active plant cells and constitutes the sole food source for the developing nematode. Although infective juveniles produce a range of cell-wall-degrading enzymes, the highly coordinated and selective incorporation of root cells, followed by their hypertrophy, makes it unlikely that nematode cell-wall-modifying proteins are used for syncytium proliferation. In the interaction between the beet cyst nematode (H. schachtii) and Arabidopsis, a gradual widening of plasmodesmata between the IFC and neighboring 
cells was the starting point for protoplast fusion, and it was suggested that plant EGase activity was involved in the observed cell wall dissolution (Grundler et al. 1998).

The hypothesis that plant cell-wall-modifying proteins are recruited by parasitic cyst nematodes is supported by gene expression surveys showing a local upregulation of plant EGases (Goellner et al. 2001), pectin methylesterases (Vercauteren et al. 2002), expansins (Wieczorek et al. 2006), and extensin (Niebel et al. 1993). Goellner and associates (2001) showed a local upregulation of five EGase genes of plant origin in tobacco cyst nematode-infected Nicotiana tabacum roots. Plant EGases are enzymes that catalyze endo-cleavage of $\beta$-1,4-glucan linkages and, thus, modify the hemicellulose-cellulose network of the cell wall (Brummell et al. 1994). In this report, we studied EGase recruitment by the potato cyst nematode G. rostochiensis in tomato. This host plant harbors at least eight EGases. Here, we demonstrate that only two isoforms, $\mathrm{Sl}$-cel7 and Sl-cel9C1, are upregulated in young expanding syncytia. We tested dynamic changes in spatial and temporal expression patterns of nematode-induced EGase genes at transcript and protein levels during syncytium development. Our results show that both the relevant transcripts and proteins are present in or very near to the developing syncytium. The importance of EGase recruitment by potato cyst nematodes for their pathogenicity was assessed by post-transcriptional gene silencing. Under in vitro conditions, silencing of St-cel7 or St-cel9C1 resulted in a severe reduction of the number of juveniles that developed into females, and a sharp increase in the fraction of abnormally developed females. Our findings suggest that local recruitment of two functionally distinct host plant EGases is essential for proper development of cyst nematodes.

\section{RESULTS}

\section{Endoglucanase expression}

in nematode-infected susceptible tomato roots.

To see whether the transcription of tomato EGases in susceptible roots is affected by infection with potato cyst nematodes, mRNA levels of individual EGases were determined in nematode-infected and uninfected root segments using semiquantitative reverse-transcription polymerase chain reaction (RT-PCR). Family member-specific primers were designed (Supplementary Table S1) and ubiquitin expression levels were determined in parallel to normalize the EGase expression data. Due to the fact that the cDNA sequence of Sl-cel6 is only partially known, no specific primers were designed to monitor the expression of this gene. Whereas expression levels of EGases were relatively low in young tomato root segments, transcripts of five of the seven EGase genes under investigation were detected in infected root tissue at 7 days postinoculation (dpi) (Fig. 1). Sl-cell and Sl-cel5 were not detected in uninfected roots in root segments containing syncytia. Two members of the EGase gene family, namely $\mathrm{Sl}$ cel7 and Sl-cel9C1, were specifically upregulated in nematode-infected roots.

\section{Spatial and temporal distribution} of Sl-cel7 and Sl-cel9C1 transcripts.

Based on the RT-PCR results, the spatial and temporal distribution of transcripts from the tomato EGases $\mathrm{Sl}$-cel7 and $\mathrm{Sl}$ cel9C1 were investigated by in situ hybridization (Fig. 2A to L). Neither Sl-cel7 (Fig. 2A) nor Sl-cel9Cl (Fig. 2C) transcripts could be detected in noninfected tomato roots. The expression of $\mathrm{Sl}$-cel7 was upregulated in young syncytial elements from 1 dpi onward (data not shown) and maintained high transcript level at 5 dpi (Fig. 2E). There was no visible fluorescent signal in the cells surrounding the young proliferating feeding site. In older syncytia (10 dpi), a high $\mathrm{Sl}$-cel7 transcripts level was observed only in the distal parts of the syncytium (= cells recently incorporated into the syncytium) and in cells directly next to the developing syncytium (Fig. 2I).

Sl-cel9C1 expression consistently followed Sl-cel7 expression and was observed from 5 dpi onward (Fig. $2 \mathrm{G}$ and $\mathrm{K}$ ). At $5 \mathrm{dpi}$, Sl-cel9Cl was expressed preferentially in the terminal syncytial elements and in parenchymatous vascular cylinder cells surrounding the syncytium (Fig. 2G). Only a weak signal was present in the IFC and in the cortex-derived part of the syncytium. In situ localization of $\mathrm{Sl}$-cel $9 \mathrm{Cl}$ transcripts in older stages of the infection process (10 dpi) revealed their presence in the cytoplasm of distal syncytial elements and in the cells neighboring the syncytium (Fig. 2K). However, the Sl-cel9C1 transcript level was higher in the adjacent cells than in syncytial elements. The hybridization with sense probes for $\mathrm{Sl}$ cel7 and Sl-cel9C1, respectively, did not give any signal on the sections of syncytia (Fig. 2B, D, F, H, J, and L).

\section{Localization of Sl-CEL7 and SI-CEL9C1 in developing syncytia.}

Antibodies against distinctive parts of S1-CEL7 and S1CEL9C1 (Supplementary Figure S1) were raised in chicken and, after purification, polyclonal IgY-s were checked for specificity using Western blot analysis (data not shown). Preimmune serum showed no reactivity with proteins extracted from syncytia and uninfected roots (data not shown) and did not give any labeling on sections (Fig. 3A). Labeling with anti-Sl-CEL7 and anti-Sl-CEL9C1 antibodies revealed a highly syncytium-specific signal. In the case of S1-CEL7, a fluorescent signal was observed already in 2-day-old syncytia (Fig. 3B). The strong labeling also was present in 5and 10-day-old syncytia (Fig. 3C and D, respectively), and it was restricted to paramural cytoplasm and to syncytial areas with dense cytoplasm. Notably, the labeling was most intense in distal syncytial elements located inside the vascular cylinder. Although its distribution pattern was similar, S1-CEL9C1 appeared later compared with S1-CEL7. In 2-day-old syncytia, no signal for S1-CEL9C1 was yet observed (Fig. $3 E)$. Specific labeling in the syncytium was observed first in distal syncytial elements from 5 dpi onward (Fig. 3F). In older syncytia (10 dpi), immunolabeling with anti-SlCEL9C1 antibody showed a similar pattern and intensity, and weak labeling also appeared in some parenchymatous vascular cylinder cells next to syncytia (Fig. 3G).

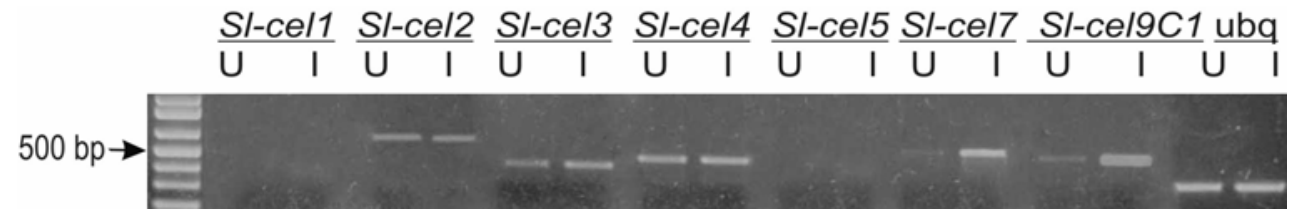

Fig. 1. Semiquantitative reverse-transcription polymerase chain reaction (RT-PCR) analysis of tomato endo- $\beta$-1,4-glucanase (EGase) transcripts in roots. RT-PCR products of seven tomato EGases were amplified from corresponding uninfected (U) and potato cyst nematode-infected (7 days postinoculation, I) root segments. Ubiquitin transcript levels were used to normalize the data. 
Identification of cel7 and cel9C1 sequences of potato.

In order to assess the roles of cel7 and cel9Cl in feeding site development by the potato cyst nematode, we decided to silence the individual genes. To assess whether the same silencing constructs could be used effectively in both tomato and potato (because they are phylogenetically closely related), we investigated the degree of relatedness between cel7 and cel9C1 in tomato and potato. Public expressed sequence tag (EST) databases were used as a starting point, because the relevant homologs were not available for potato.

First, potato EST databases were screened for the presence of potential orthologous EST of Sl-cel7 and Sl-cel9C1. The first screen resulted in two EST, CK248726 (966 bp; 95\% identical to Sl-cel7) and CK248727 (426 bp; 94\% identical to Sl-cel7). Remarkably, the screen for $\mathrm{Sl}$-cel9C1 homologs resulted in two distinct potato unigenes, SGN-U286389 (pre- dicted peptide of 102 amino acids [aa], carbohydrate binding module [CBM], and part of a linker with $60 \%$ identity to CBM of S1-CEL9C1) and SGN-U272121 (predicted peptide of 403 aa; major part of catalytic domain, linker, and CBM with $93 \%$ identity to S1-CEL9C1).

In order to establish the degree of relatedness between $\mathrm{Sl}$ cel7 and St-cel7, a genome walking strategy was followed (Siebert et al. 1995) on the basis of genomic DNA extracted from in vitro cultured potato plants (line 6487-9) (Horsman et al. 2001). Primer design was based on tomato (Y11268) and tobacco (AF362947) cel7 cDNAs. Following standard procedures, the gDNA of St-cel7 was sequenced, and in silico splicing and translation resulted in a predicted protein with $95 \%$ identity to $\mathrm{Sl}$-cel7. Hence, it is feasible to design a cellulase family member-specific double-stranded (ds)RNA construct that most probably will silence both $\mathrm{Sl}$-cel7 and St-cel7.
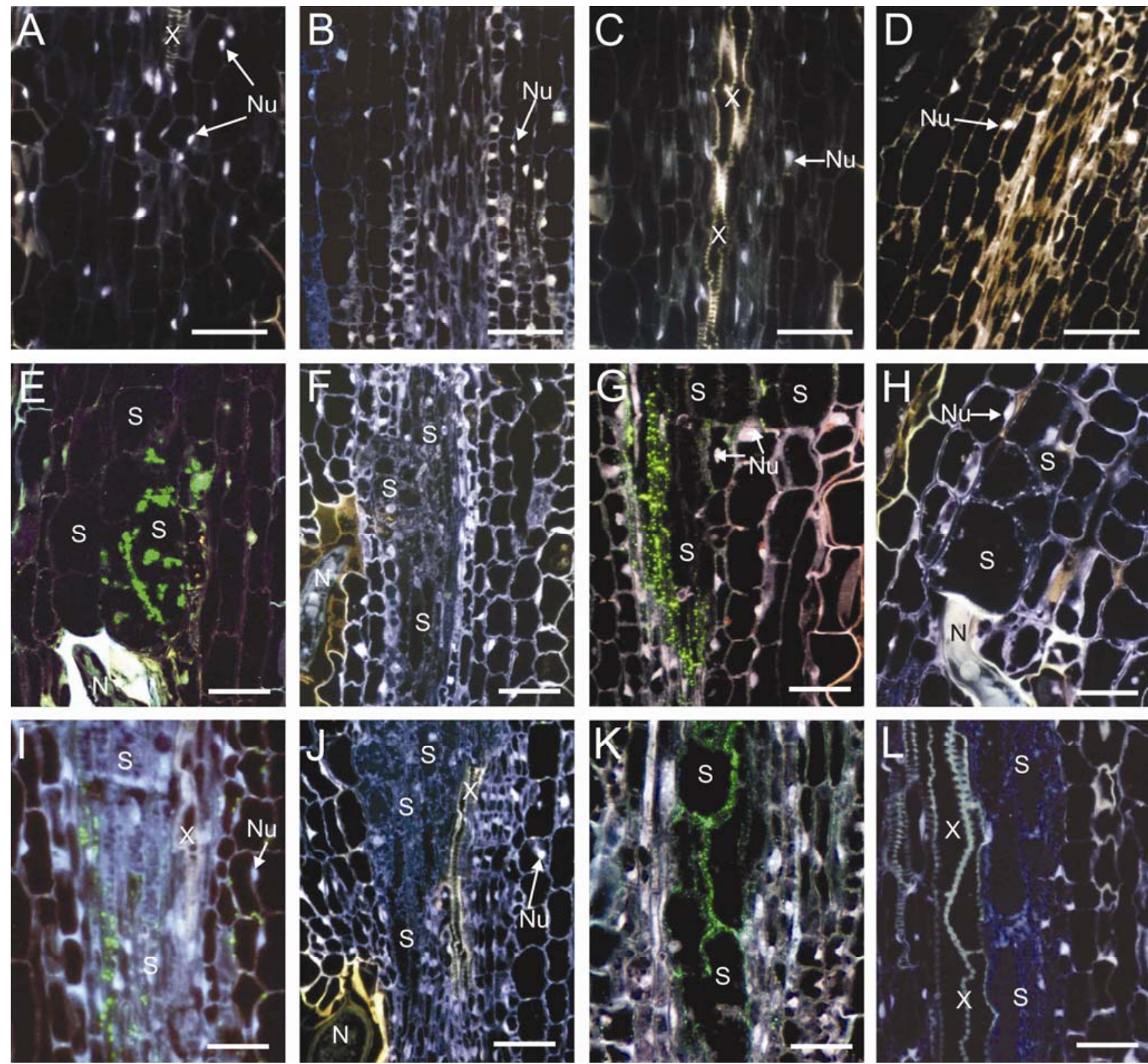

Fig. 2. In situ detection of $\mathbf{A}, \mathbf{B}, \mathbf{E}, \mathbf{F}, \mathbf{I}$, and $\mathbf{J}$, Sl-cel7 and $\mathbf{C}, \mathbf{D}, \mathbf{G}, \mathbf{H}, \mathbf{K}$, and $\mathbf{L}$, Sl-cel $9 \mathrm{Cl}$ transcripts on longitudinal sections of the susceptible tomato cv Moneymaker roots infected with potato cyst nematode. Hybridization signal is visible as green coloration due to FITC fluorescence. Sections through uninfected roots hybridized with A, Sl-cel7 antisense probe; B, Sl-cel7 sense probe; C, Sl-cel9C1 antisense probe; and D, Sl-cel9C1 sense probe. Sections through syncytia (5 days postinoculation [dpi]) hybridized with E, Sl-cel7 antisense probe; F, Sl-cel7 sense probe; $\mathbf{G}, \mathrm{Sl}$-cel $9 \mathrm{Cl}$ antisense probe; and $\mathbf{H}$, Slcel9Cl sense probe. Sections through syncytia (10 dpi) hybridized with I, Sl-cel7 antisense probe; J, Sl-cel7 sense probe; K, Sl-cel9C1 antisense probe; and $\mathbf{L}, \mathrm{Sl}$-cel $9 \mathrm{Cl}$ sense probe. $\mathrm{N}=$ nematode, $\mathrm{Nu}=$ nucleus, $\mathrm{S}=$ syncytium, and $\mathrm{X}=\mathrm{xylem}$. Bars: $30 \mu \mathrm{m}$. 
The remarkable finding of a unigene that was predicted to code for a CBM domain related to the CBM49 family member that was present in Sl-cel9Cl (because it contains two conserved exposed tryptophan residues that are thought to be involved in cellulose binding) (Urbanowicz et al. 2007) prompted us to investigate whether this CBM indeed was linked to a family 9 glycosyl hydrolase. Again, a genome walking strategy was followed using gDNA isolated from line 6487-9 in vitro potato plants. Primer design was based on tobacco (AF362949) and tomato (AF098292) cel9C1 (formerly called cel8) cDNAs. This procedure resulted in the identification of a major part of the genomic sequence of a second homolog of Sl-cel9Cl. The catalytic domain of the nearly complete predicted protein was 92 and $83 \%$ identical to the corresponding domains in tobacco and tomato. Hence, potato harbors at least two class $\mathrm{C}$ family 9 glycosyl hydrolases that differ mainly in linker and CBM. It is concluded that a dsRNA construct designed to silence $\mathrm{Sl}$ cel9C1 and St-cel9C1 probably will not interfere with the expression of a second homolog in potato, here provisionally labeled as St-cel9C2.

\section{Post-transcriptional silencing of cel7 and cel9C1,} and effects on nematode growth and development.

Attempts to silence $\mathrm{cel} 7 \mathrm{and}$ cel9C1 in tomato failed in the case of the latter. This observation, together with the fact that no fragments of a second tomato cel9 could be found among the $>100,000$ EST available in public data bases (A. Karczmarek, unpublished results) suggest that tomato harbors a single class $\mathrm{C}$ EGase. Because we wanted to test the relevance of both genes in a single plant background, and keeping in mind that potato presumably harbors a second, somewhat distinct class C EGase, we decided to do the silencing experiments in potato. To this end, we transformed potato with Sl-cel7 (360 bp) and Sl-cel9Cl (379 bp) hairpin constructs. After Agrobacterium spp.-mediated potato transformation, six and eight independent lines harboring cel7 or cel9Cl hairpin constructs, respectively, were regenerated. The silencing efficiency was verified by RT-PCR experiments and strong suppression of the target genes was found in three hp-Sl-cel7 lines $(s 7.1, s 7.2$, and s7.3) and in three hp-Slcel9C1 lines ( $s 9$ C.1, $s 9$ C.2, and $s 9$ C.3) (Fig. 4A). Apart from silencing of the target St-cel9C1 gene, line s9C.2 showed silencing of St-cel7 gene. Two potato lines with abnormal phenotype, $n s 7.4$ and $n s 9$ C.4, showed no reduction in St-cel7 and St-cel9C1 transcript levels. Plants of $n s 7.4$ and $n s 9$ C.4 lines were dwarf and their root systems were poorly developed. Except for occasional transformation effects, no obvious silencing-related aberrant phenotypes were observed for the remaining lines.

Effect of silencing on potato cyst nematode development was tested in vitro. Silenced potato plants and the corresponding empty vector controls were in vitro inoculated with potato cyst nematodes. Five weeks after inoculation, nematode development in EGase-silenced plants was compared with their development on control plants. Two parameters were scored: i) the total number and ii) the phenotype of adult females. On control plants, the total number of $\mathrm{J} 2 \mathrm{~s}$ that developed into females was $136 \pm 14$ (average \pm standard error) (Fig. 4B, gray and black bars taken together). On silenced plants with a normal phenotype, the largest reductions in total number of females ranged from $60 \%$ in cel7-silenced potato plants (line s7.1; 55 [11 females] to $31 \%$ in cel9C1-silenced plants [line s9C.2 (this line is silenced for both EGases)]; 94 [4 females]) (Fig. 4B). Microscopic observations showed that, in most cases,
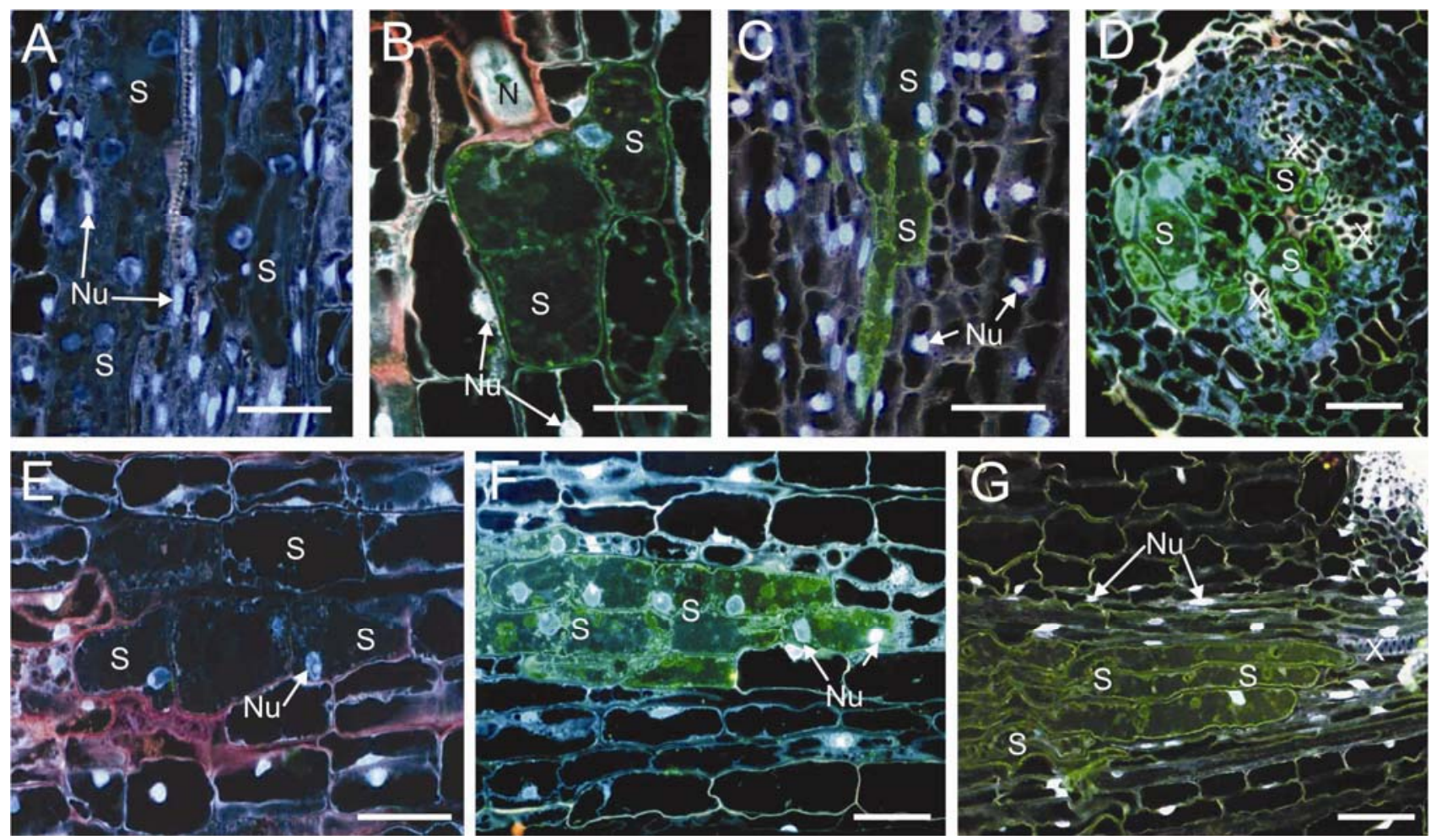

Fig. 3. Immunofluorescent detection of $\mathbf{B}$ through D, S1-CEL7 and $\mathbf{E}$ through $\mathbf{G}$, S1-CEL9C1 in syncytia induced in susceptible tomato cv. Moneymaker roots by potato cyst nematodes. Immunolocalization signal is visible as green coloration due to Alexa Fluor 488 fluorescence. Blue coloration of nuclei is due to counterstaining with 4',6-diamidino-2-phenyliadole. A, Longitudinal section through syncytium (10 days postinoculation [dpi]) probed with preimmune serum; B, longitudinal section of syncytium ( 2 dpi) probed with Sl-CEL7 antiserum; C, longitudinal section through distal part of syncytium (5 dpi) probed with Sl-CEL7 antiserum; D, cross section of syncytium (10 dpi) probed with Sl-CEL7 antiserum; E, longitudinal section of syncytium (2 dpi) probed with S1-CEL9C1 antiserum; F, longitudinal section through distal part of syncytium (5 dpi) probed with S1-CEL9C1 antiserum; and G, longitudinal section through distal part of syncytium (10 dpi) probed with Sl-CEL9C1 antiserum. $\mathrm{N}=$ nematode, $\mathrm{Nu}=$ nucleus, $\mathrm{S}=$ syncytium, and $\mathrm{X}=\mathrm{xylem}$. Bars: $30 \mu \mathrm{m}$ for $\mathrm{A}$ through $\mathrm{C}, \mathrm{E}$, and $\mathrm{F}$ and $60 \mu \mathrm{m}$ for $\mathrm{D}$ and $\mathrm{G}$. 
nematode development stopped at the $\mathrm{J} 2$ to $\mathrm{J} 3$ life stage (Fig. 4F and $\mathrm{G}$ ). Under normal conditions, the posterior part of the female body was spherical and opaque (Fig. 4C) because it was filled with eggs. On EGase-silenced plants, the percentage of fully developed females had dropped by 89 and $78 \%$ in line $s 7.1$ and $s 9$ C. 1 , respectively ( $15 \pm 4$ and $29 \pm 13$ fully developed females). Poorly developed females were smaller, and the posterior part of their body was transparent and saccate (Fig.
4D and E) because they did not contain eggs. Growth on EGasesilenced potato plants resulted in a sharp increase of the number of poorly developed females $(40 \pm 19$ for $s 7.1$ and $82 \pm 28$ for $s 9.1$ compared with $5.5 \pm 2$ in control) (Fig. 4B, black bars). Hence, the post-transcriptional silencing of St-cel7 or Stcel9C1 resulted in potato in a reduced number of female nematodes, and a major percentage of the females that could develop showed a severely aberrant phenotype.

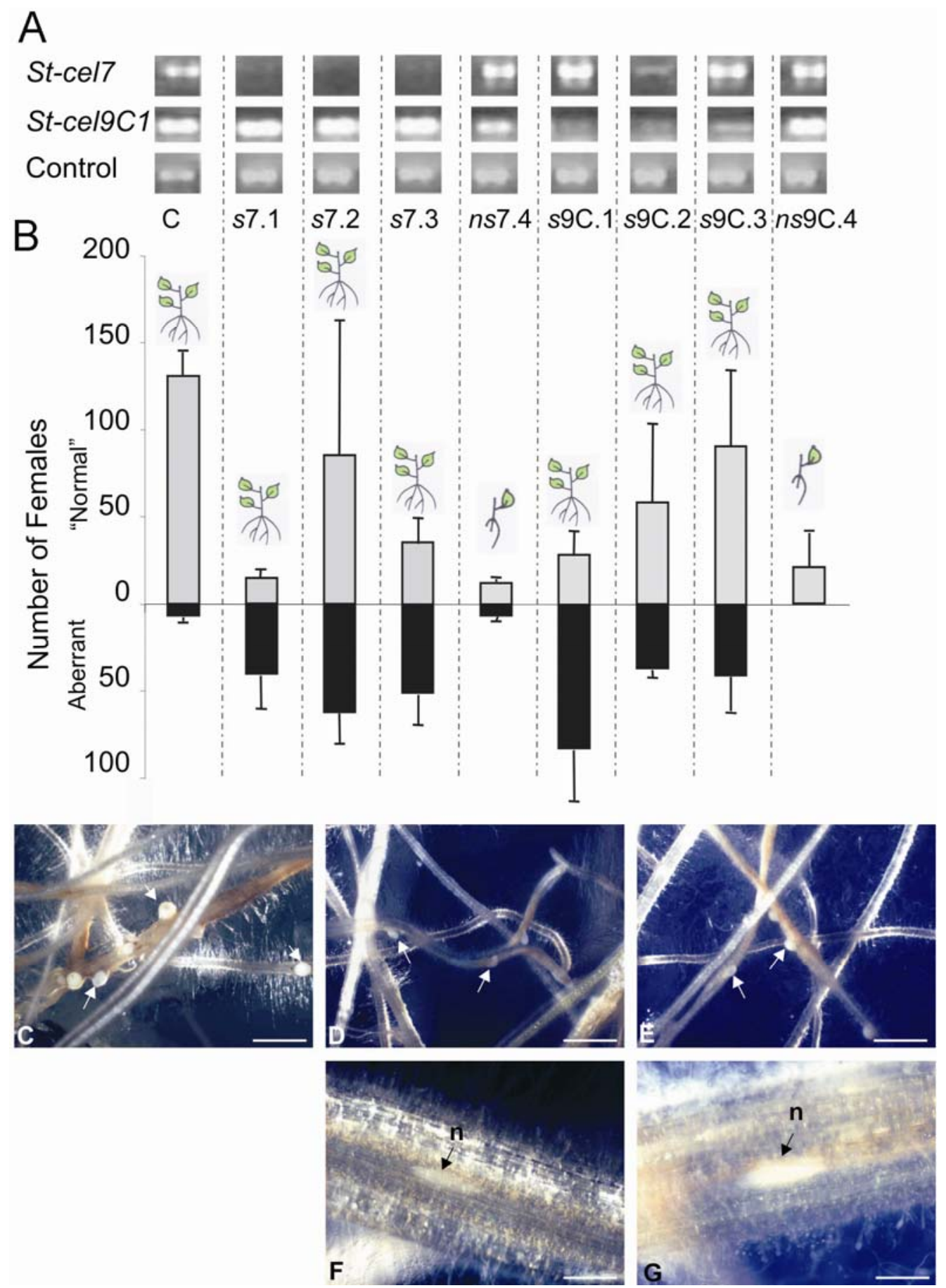

Fig. 4. Effect of dsRNA-based endo- $\beta$-1,4-glucanase silencing on the development of potato cyst nematode. A, Semiquantitative reverse-transcription polymerase chain reaction analysis of expression of $\mathrm{St}$-cel7, St-cel9C1, and leucine-rich repeat-containing potato gene in infected control potato plants (C) and transgenic or silenced potato lines $n s 7, s 7.1-s 7.3, n s 9$ C, and $s 9$ C.1-s9C.3. B. Average numbers (+standard error) of normal females (spherical and opaque; gray bars) and aberrant females (saccate and transparent, black bars) developed on control and silenced plant lines. Plant phenotype is indicated as pictograms above the bars. C, Normally developed adult females (arrows) on control plant at 5 weeks after inoculation; D, poorly developed females (arrows) on St-cel7-silenced potato plant at 5 weeks after inoculation; E, poorly developed females (arrows) on St-cel9C1-silenced potato plant at 5 weeks after inoculation; $\mathbf{F}$, juvenile (arrow) arrested in the parasitic J2/J3 stage on St-cel-silenced potato plant at 5 weeks after inoculation; and $\mathbf{G}$, juvenile (arrow) arrested in the parasitic J2/J3 stage on St-cel9C1-silenced potato plant at 5 weeks after inoculation. Bars: $5 \mathrm{~mm}$ for C through E and $1 \mathrm{~mm}$ for F and G. 


\section{DISCUSSION}

We sought to identify plant EGases that are locally upregulated as a result of infection by the potato cyst nematode $\mathrm{G}$. rostochiensis, to investigate the spatial and temporal distribution of the relevant transcripts and proteins, and to assess the importance of these plant proteins for the development of this parasitic nematode. We found two EGases, Sl-cel7 and Slcel9C1, that are successively upregulated in and immediately around the developing feeding site induced by the nematode. Silencing of Sl-cel9Cl appeared to be incompatible with the regeneration of viable tomato plants and, therefore, homologs of $\mathrm{cel} 7 \mathrm{and}$ cel9C1 were silenced in potato, a close relative. In vitro phenotypes of potato plants silenced for either of these EGases apparently were normal, and this manipulation resulted in a severely hampered growth and development of potato cyst nematodes. Remarkably, other plant EGases expressed in the nematode infection zone, cel2, cel3, and cel4, did not functionally replace $\mathrm{cel} 7 \mathrm{\text {or }}$ cel9C1.

\section{Why do cyst nematodes specifically recruit cel7 and cel9C1?}

In the interaction between Arabidopsis thaliana and the beet cyst nematode $H$. schachtii, a strong activation of DR5, an artificial auxin responsive promoter, inside the initial feeding cells as early as $18 \mathrm{~h}$ postinoculation was observed (Karczmarek et al. 2004). This finding specified earlier results that point at an essential role of auxin in the induction of feeding sites by cyst and root knot nematodes in a range of host plants (Goverse et al. 2000; Hutangura et al. 1999). Among the currently known members of the tomato EGase family, only Sl-cel7 was shown clearly to be auxin inducible (Catalá et al. 1997). Without exogenous auxin application, the Sl-cel7 mRNA abundance was very low in tomato hypocotyls. Hence, the early induction of Sl-cel7 could be the result of the accumulation of auxin in the cyst-nematode-induced initial feeding site. It is noted that $N t$ cel7-an auxin-inducible homolog of Sl-cel7 from tobaccoresided among the five EGases that were reported to be upregulated in tobacco roots infected by G. tabacum subsp. solanacearum (Goellner et al. 2001).

Sl-cel9C1 (formerly indicated as TomCel8) originally was isolated from tomato hypocotyls (Catalá and Bennett 1998) and, so far, it is the only tomato EGase that harbors a CBM. This CBM belongs to a relatively new CBM family, namely CBM49, and this module was shown to bind to crystalline cellulose (Urbanowicz et al. 2007). Our inability to regenerate viable $S l$-cel9C1-silenced tomato plants could be explained by hypothesizing that $\mathrm{Sl}$-cel9Cl is essential for plant development and that tomato does not harbor a functionally similar gene. The finding that potato possesses multiple EGases with distinct CBM may explain why it was relatively straightforward to generate St-cel9C1-silenced potato plants (our dsRNA target region included a part of the linker region and the CBM). Presumably, essential functions of St-cel9C1 were taken over by the other potato $c e l 9 \mathrm{C}$, here provisionally labeled as Stcel9C2. Among higher plants, the presence of multiple distinct EGases with a family $49 \mathrm{CBM}$ is not unusual because both rice (Oryza sativa) and A. thaliana harbor (at least) three CEL9 isoforms with a CBM49 (BAF04337, BAF04335, BAF16834, AAF69707, AAC35539, and AAG51703).

Syncytium formation by potato cyst nematodes involves local degradation of the plant cell wall. The primary cell wall of dicotyledons (type I) contains high amounts of xyloglucan that interlocks the cellulose microfibrils. Specific recruitment of a cel7 and a cel9C EGase by the potato cyst nematode could be sufficient to promote the (local) degradation of xyloglucan and noncrystalline cellulose (CEL7), and crystalline cellulose
(CEL9C). The severe impact of EGase silencing in potato on cyst nematode development shows that parasitism is not just accompanied by the local activation of plant EGases. It is concluded that successful plant parasitism by potato cyst nematodes requires the local upregulation of at least two distinct kinds of plant EGases.

\section{Functional redundancy among plant EGases.}

Strong indications for functional redundancy among tomato EGases were generated by Lashbrook and associates (1998) and Brummell and associates (1999). In an attempt to inhibit the softening of tomato fruit, the authors inhibited the accumulation of tomato EGase Sl-cell and Sl-cel2 transcripts by using an antisense approach. Although suppressed lines showed a 99\% decrease in mRNA levels, no change in the ripening behavior of the tomato fruit was observed. Fruit ripening in tomato involves at least three EGase family members, Sl-cell, Sl-cel2, and Sl-cel5 (all GH9 class B members), and the absence of an effect of strongly reduced mRNA levels on fruit softening suggests functional redundancy among these EGases (Brummell and Harpster 2001).

Keeping the functional overlap between tomato cellulases in mind, the severe impact of both cel7 and cel9C silencing in potato on cyst nematode development as observed in our experiments was unexpected. Cel7 belongs to class B of the family 9 glycosyl hydrolases (class B: a catalytic domain preceded by a signal sequence for secretion). Two other GH9 class B members (cel2 and cel4) also were expressed in tomato roots (Fig. 1). Provided that homologs Sl-cel2 and Sl-cel4 are present in potato, it

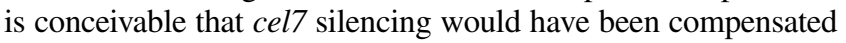
by these two other GH9 class B members. Similarly, we have shown that potato harbors at least two distinct GH9 class C members (St-cel9C1 and "St-cel9C2"). Our results suggest that potato is relatively flexible in replacing silenced EGases. Apparently, the parasite $G$. rostochiensis cannot compensate for a lack of CEL7 activity by recruiting other, functionally similar plant cellulases such as CEL2, CEL3, or CEL4.

\section{MATERIALS AND METHODS}

\section{Plant material and nematode inoculation.}

Seed of the potato cyst nematode-susceptible tomato (Solanum lycopersicum L. cv. Moneymaker) were surface sterilized with $0.8 \%$ commercial bleach (vol/vol) for $25 \mathrm{~min}$ and rinsed three times in sterile tap water. Seed (five per plate) were germinated in a petri dish (diameter $=9 \mathrm{~cm}$ ) containing $1.5 \%(\mathrm{wt} / \mathrm{vol})$ water agar in the dark at $18^{\circ} \mathrm{C}$. Germinating seed were transferred into petri dishes containing Gamborg's B5 medium supplemented with $0.2 \%$ (wt/vol) sucrose and $0.3 \%$ (wt/vol) Gelrite (Duchefa, Haarlem, The Netherlands). Tomato plants were grown on slightly tilted plates at $18^{\circ} \mathrm{C}$ under 16- and 8-h light and dark cycles, respectively. Potato plants (S. tuberosum L. line 6487-9) (Horsman et al. 2001) were grown on Murashige and Skoog basal medium containing 2\% (wt/vol) sucrose and $0.8 \%(\mathrm{wt} / \mathrm{vol})$ Daishin agar (Brunschwig Chemie BV, Amsterdam) at $24^{\circ} \mathrm{C}$ under 16 - and 8-h light and dark cycles, respectively. Surface-sterilized preparasitic J2s from the cyst nematode G. rostochiensis Woll. (pathotype Ro1, Mierenbos) were prepared as described by Goverse and associates (2000). Approximately $10 \mathrm{~J} 2 \mathrm{~s}$ per root tip were transferred to 10- to 12-day-old tomato or potato plants using sterile siliconized pipette tips. For the nematode resistance test of the transgenic potato plants, nine plants (three plants per petri dish) of each line were inoculated as described above. After inoculation, plants were kept in the dark at $18^{\circ} \mathrm{C}$. Five weeks after infection, the number of adult females per petri dish was determined and the phenotype of the females was assessed. 


\section{Semiquantitative RT-PCR.}

At $7 \mathrm{dpi}$, tomato root segments (length approximately $1 \mathrm{~cm}$ ) from both infected and noninfected roots were collected and frozen in liquid $\mathrm{N}_{2}$. For total RNA isolation, 50 to $100 \mathrm{mg}$ of frozen root material was incubated in $0.75 \mathrm{ml}$ of TRIzol reagent (Gibco/Life Technologies, Breda, The Netherlands). Glass beads (425 to $600 \mu \mathrm{m}$ ) were used to homogenize the segments in a Silamat S5 shaker (Vivadent, Austria). Oligo $(\mathrm{dT})_{25}$ Dynabeads (Dynal, Oslo, Norway) were used to isolate mRNA. For first-strand cDNA synthesis, the GeneAmp RNA PCR kit (Applied Biosystems, Foster City, CA, U.S.A.) was used. On the basis of the alignment of cDNA sequences of eight known tomato EGases (Sl-cell U13054, Sl-cel2 U13055, Sl-cel3 U78526, Sl-cel4 U20590, Sl-cel5 AF077339, Sl-cel6 Q9S946 [partial sequence], Sl-cel7 Y11268, and Sl-cel9C1 AF098292), specific primers were designed. Individual EGase transcripts were amplified in AmpliTaq Gold DNA polymerasedriven PCR reactions (Applied Biosystems). The following profile was used: $95^{\circ} \mathrm{C}$ for $10 \mathrm{~min}$ and 39 subsequent cycles of $30 \mathrm{~s}$ at $94^{\circ} \mathrm{C}, 45 \mathrm{~s}$ at $53^{\circ} \mathrm{C}$, and $45 \mathrm{~s}$ at $72^{\circ} \mathrm{C}$. Products were analyzed on a $1.2 \%(\mathrm{wt} / \mathrm{vol})$ agarose gels.

\section{Plant material for in situ examination.}

Noninfected root segments and root segments containing 5and 10-day-old syncytia were excised and fixed in 4\% (wt/vol) paraformaldehyde in $50 \mathrm{mM}$ PIPES [piperazine- $N, N^{\prime}$-bis(2ethanesulfonic acid)] buffer ( $\mathrm{pH}$ 6.9) for $2 \mathrm{~h}$ at room temperature. After washing the segments four times for $30 \mathrm{~min}$ in the same buffer, they were dehydrated and embedded in butylmethyl-methacrylate (BMM) resin (de Almeida Engler et al. 2001). Embedded material was serially cut into semithin (2 $\mu \mathrm{m}$ thick) sections with a Leica RM2165 microtome. The sections were adhered to Superfrost 3-chamber slides (MJ Research, Watertown, MA, U.S.A.) on a slide heating plate $(2 \mathrm{~h}$ at $50^{\circ} \mathrm{C}$ ). For further experimentation, the BMM resin was removed and sections were rehydrated, digested with Proteinase K (Sigma, St. Louis), and dehydrated again.

\section{In situ hybridization in tomato root sections.}

Digoxigenin (DIG)-labeled single-stranded (ss)-cDNA probes for Sl-cel7 and Sl-cel9Cl were prepared in two successive amplification reactions (Expand High Fidelity PCR System, Roche) using primer pairs Sl-cel7-A or Sl-cel9C1-A. In the first reaction, ds-cDNA fragments were generated by one of the primer pairs using a TOPO vector harboring cDNA of $\mathrm{Sl}$-cel7 or $\mathrm{Sl}$-cel9Cl as template. In the second reaction, a PCR DIG probe synthesis kit (Roche, Branchburg, NJ, U.S.A.) was used to synthesize antisense or sense ss-cDNA probes with 2 $\mu \mathrm{l}$ of the first PCR product as a template and $2 \mu \mathrm{M}$ of a single primer. Both reactions were performed on a MJ Research PTC-100 thermal cycler using the following PCR profile: $95^{\circ} \mathrm{C}$ for $2 \mathrm{~min} ; 35$ subsequent cycles of $15 \mathrm{~s}$ at $95^{\circ} \mathrm{C}, 30 \mathrm{~s}$ at $54^{\circ} \mathrm{C}$, and $45 \mathrm{~s}$ at $72^{\circ} \mathrm{C}$; followed by $7 \mathrm{~min}$ at $72^{\circ} \mathrm{C}$. Unincorporated nucleotides were removed with a Mini Quick Spin DNA columns (Roche). Both amplification products and purification steps were verified on agarose gel.

For in situ hybridizations, frame-seal incubation chambers (MJ Research) were attached to slides with root sections pretreated as described above. Into each slide-well, $20 \mu \mathrm{l}$ of hybridization mix was pipetted. This mix consisted of $50 \%$ (vol/vol) formamide, 10\% (wt/vol) dextran sulfate, tRNA at $250 \mathrm{ng} / \mathrm{ml}$ (Sigma), Poly(A) at $100 \mu \mathrm{g} / \mathrm{ml}$ (Sigma), $0.3 \mathrm{M}$ $\mathrm{NaCl}, 0.01 \mathrm{M}$ Tris- $\mathrm{HCl}$ (pH 7.5), 1 mM EDTA, 1× Denhard's solution (Amersham Pharmacia, Uppsala, Sweden), $0.01 \mathrm{mM}$ DTT and DIG-labeled ss-cDNA antisense or sense probe at 2 $\mu \mathrm{g} / \mathrm{ml}$. Chambers were sealed and kept overnight at $45^{\circ} \mathrm{C}$. Afterward, sections were washed in $2 \times \mathrm{SSC}$ buffer $(1 \times \mathrm{SSC}$ is
$0.15 \mathrm{M} \mathrm{NaCl}$ plus $0.015 \mathrm{M}$ sodium citrate) ( $\mathrm{pH} 7.2)$ for $25 \mathrm{~min}$ at room temperature, followed by three washes with $0.2 \times \mathrm{SSC}$ at $53^{\circ} \mathrm{C}$ for $25 \mathrm{~min}$ each. Finally, slides were rinsed three times with sterile distilled water for $1 \mathrm{~min}$ and subjected to immunodetection of DIG, which was carried out with a Fluorescent antibody enhancer set for DIG detection (Roche). After air drying in the darkness slides were mounted with ImmunoFluore mounting medium (ICN). Slides were viewed using an Olympus AX70 "Provis" microscope equipped with a UM61002 fluorescence filter set.

\section{Antibody production.}

Based on the alignment of eight tomato EGases, specific fragments for Sl-cel7 and Sl-cel9C1 were amplified using primer pairs $S l$-cel7-B and Sl-cel9Cl-B. Isozyme-specific protein fragments were produced in a $\mathrm{pBAD} /$ Thio-TOPO expression system (Invitrogen, San Diego, CA, U.S.A.) and recombinant fragments were purified on a Ni-NTA spin column (Qiagen, Valencia, CA, U.S.A.). Purified antigen (approximately $50 \mu \mathrm{g}$ ) was injected into chicken, and eggs were collected after 4 weeks. IgY-s were precipitated from the yolk by PEG 6000 and affinity-purified on a HiTrap IgY column (Amersham/ Pharmacia). Dialyzed fractions of anti-Sl-CEL7 and anti-S1CEL9C1 were used for immunolocalization studies.

\section{Tissue preparation and immunocytochemical localization.}

Root segments with syncytia at 2, 5, and 10 dpi were fixed in $2 \%$ (vol/vol) glutaraldehyde and $2 \%$ (wt/vol) paraformaldehyde in $0.1 \mathrm{M}$ phosphate-buffered saline (PBSO $(\mathrm{pH} \mathrm{7.0)}$ for $2 \mathrm{~h}$, washed four times with PBS, and embedded in BMM resin. Sections ( $3 \mu \mathrm{m}$ thick) were collected on silane-coated slides and the resin was removed (de Almeida Engler et al. 2001). After rehydration in PBS, unspecific epitopes were blocked by incubation for $1 \mathrm{~h}$ in PBS supplemented with 5\% (wt/vol) nonfat dried milk and $0.05 \%$ (vol/vol) Tween 20 . Thereafter, slides were treated with primary antibodies (anti-Sl-CEL7 or anti-Sl-CEL9C1) diluted 1:10 or 1:20 in PBS supplemented with $1 \%$ (wt/vol) nonfat dried milk and $0.05 \%$ (vol/vol) Tween $20\left(1 \mathrm{~h}\right.$ at $\left.37^{\circ} \mathrm{C}\right)$. Subsequently, slides were washed twice in PBS with $0.1 \%$ (vol/vol) Tween 20 and once in PBS. In a next step, sections were incubated in goat anti-chicken immunoglobulin $\mathrm{G}$ at $5 \mu \mathrm{g} / \mathrm{ml}$ conjugated with Alexa Fluor 488 (Molecular Probes, Eugene, OR, U.S.A.) in PBS $\left(1 \mathrm{~h}\right.$ at $\left.37^{\circ} \mathrm{C}\right)$ and washed three times in PBS as described above. Samples were counterstained with 4',6-diamidino-2-phenyliadole at $1 \mu \mathrm{g} / \mathrm{ml}$ for $10 \mathrm{~min}$ and $0.01 \%$ (wt/vol) toluidine blue for $1 \mathrm{~min}$, and mounted with ImmunoFluore mounting medium (ICN). In negative controls, primary antibodies were replaced by preimmune chicken serum. The sections were examined as described above for in situ analysis.

\section{Cloning of potato cel7 and cel9C1.}

The putative sequences of St-cel7 and St-cel9C1 were amplified from potato line 6487-9 (Horsman et al. 2001). Genomic DNA was isolated using a DNeasy Plant DNA isolation system (Qiagen) and amplified with primers designed on the basis of corresponding tomato and tobacco cDNA sequences for cel7 and cel9C1 ( cel7 $F$ and $R$ and cel9C1 $F$ and $R$ ). The amplification products with sizes of 1,350 and 1,650 bp (cel7 and cel9Cl, respectively) were cloned and sequenced. A nucleotide-nucleotide BLAST search (blastn) with the putative sequences of Stcel7 and St-cel9C1 revealed highest homology with cDNA sequences of the tomato cel7 and cel9Cl. Additional genomic sequences were identified by using a Universal GenomeWalker Kit (BD Biosciences Clontech, Allschwil, Switzerland) according to the manufacturer's instructions. Primer sequences are listed in Supplementary Table S2. PCR temperature profiles were as follows: seven cycles of $94^{\circ} \mathrm{C}$ for $2 \mathrm{~s}$ and $72^{\circ} \mathrm{C}$ for 3 
min; 32 cycles of $94^{\circ} \mathrm{C}$ for $2 \mathrm{~s}$ and $65^{\circ} \mathrm{C}$ for $3 \mathrm{~min}$; within the last cycle, the PCR samples were incubated for an additional 4 min at $65^{\circ} \mathrm{C}$. The same profiles were used for the nested PCR; however, five cycles were run with the first profile and 25 cycles with the second one. The PCR fragments were cloned into the TOPO2.1 vector for transformation of Escherichia coli (Invitrogen) and subsequently sequenced using standard procedures. The Vector NTI Advance program package (InforMax) was used for DNA sequence analysis.

\section{dsRNA constructs and plant transformation.}

Fragments of Sl-cel7 (360 bp) and Sl-cel9C1 (379 bp) were amplified from a cDNA library using primers that incorporate an $a t t B$ recombination sites ( $S l$-cel7-C and Sl-cel9C1-C). The $a t t B-$ flanked PCR products were introduced into a Gateway (Invitrogen)-compatible vector (pk7GWIWG2(I)) (Karimi et al. 2002), and expression was driven by the CaMV $35 \mathrm{~S}$ promoter which previously also was shown to be active in developing syncytia (Goverse et al. 1998). Constructs were introduced into Agrobacterium tumefaciens LBA4404 by electroporation, and positive clones were used for potato transformation. Stem segments $(0.5$ to $1 \mathrm{~cm}$ ) from in-vitro-grown plants were transformed with pk7GWIWG2::Sl-cel7 or pk7GWIWG2::Sl-cel9C1 and regenerated (van Engelen et al. 1994). Primer pairs Sl-cel7 and Slcel $9 \mathrm{Cl}$ were used to check the silencing efficiency in individual transgenic potato lines (3-week-old plants; root segment length approximately $1 \mathrm{~cm}$ ), and a cluster-specific primer set for a constitutively expressed LRR-containing potato gene was used to normalize band intensities (Bakker et al. 2003). Transgenic potato plants and empty vector-transformed controls were in vitro inoculated with nematodes as described above.

\section{ACKNOWLEDGMENTS}

Funding from the European Union (NONEMA, grant QLK5-1999$1501)$ is gratefully acknowledged.

\section{LITERATURE CITED}

Bakker, E., Butterbach, P., Rouppe van der Voort, J., van der Vossen, E., van Vliet, J., Bakker, J., and Goverse, A. 2003. Genetic and physical mapping of homologues of the virus resistance gene Rx1 and the cyst nematode resistance gene Gpa2 in potato. Theor. Appl. Genet. 106:15241531

Brummell, D. A., and Harpster, M. H. 2001. Cell wall metabolism in fruit softening and quality and its manipulation in transgenic plants. Plant Mol. Biol. 47:311-340.

Brummell, D. A., Lashbrook, C. C., and Bennett, A. B. 1994. Plant endo1,4- $\beta$-glucanases: Structure, properties and their physiological function. Am. Chem. Soc. Symp. Ser. 556:100-129.

Brummell, D. A., Hall, B. D., and Bennett, A. B. 1999. Antisense suppression of tomato endo-1,4- $\beta$-glucanase Cel 2 mRNA accumulation increases the force required to break fruit abscission zones but does not affect fruit softening. Plant Mol. Biol. 40:615-622.

Carpita, N. C., and Gibeaut, D. M. 1993. Structural models of primary cell walls in flowering plants: Consistency of molecular structure with the physical properties of the walls during growth. Plant J. 3:1-30.

Catalá, C., and Bennett, A. B. 1998. Cloning and sequence analysis of TomCel8: A new plant endo- $\beta-1,4-\mathrm{D}$-glucanase gene, encoding a protein with a putative carbohydrate binding domain (accession no. AF098292) (PGR98-209). Plant Physiol. 118:1535.

Catalá, C., Rose, J. K. C., and Bennett, A. B. 1997. Auxin regulation and spatial localization of an endo-1,4- $\beta$-D-glucanase and a xyloglucan endotransglycosylase in expanding tomato hypocotyls. Plant J. 12:417426 .

de Almeida Engler, J., De Grooth, R., Van Montagu, M., and Engler, G. 2001. In situ hybridization to mRNA of Arabidopsis tissue sections. Methods 23:325-334.

Goellner, M., Smant, G., De Boer, J. M., Baum, T. J., and Davis, E. L. 2000. Isolation of $\beta$-1,4-endoglucanase genes from Globodera tabacum and their expression during parasitism. J. Nematol. 32:154-165.

Goellner, M., Wang, X. H., and Davis, E. L. 2001. Endo- $\beta$-1,4-glucanase expression in compatible plant-nematode interactions. Plant Cell 13:2241-2255.

Goverse, A., Biesheuvel, J., Wijers, G. J., Gommers, F. J., Bakker, J., Schots, A., and Helder, J. 1998. In planta monitoring of the activity of two constitutive promoters, CaMV $35 \mathrm{~S}$ and TR2', in developing feeding cells induced by Globodera rostochiensis using green fluorescent protein in combination with confocal laser scanning microscopy. Physiol. Mol. Plant Pathol. 52:275-284.

Goverse, A. Overmars, H., Engelbertink, J., Schots, A., Bakker, J., and Helder, J. 2000. Both induction and morphogenesis of cyst nematode feeding cells are mediated by auxin. Mol. Plant-Microbe Interact. 13:1121-1129.

Grundler, F. M. W., Sobczak, M., and Golinowski, W. 1998. Formation of wall openings in root cells of Arabidopsis thaliana following infection by the plant-parasitic nematode Heterodera schachtii. Eur. J. Plant Pathol. 104:545-551.

Horsman, K., Gavrilenko, T., Bergervoet, M., Huigen D. J., Joe, A. T. W., and Jacobsen, E. 2001. Alteration of the genomic composition of Solanum nigrum (+) potato backcross derivatives by somatic hybridization: Selection of fusion hybrids by DNA measurements and GISH. Plant Breed. 120:201-207.

Hutangura, P., Mathesius, U., Jones, M. G. K., and Rolfe, B. G. 1999. Auxin induction is a trigger for root gall formation caused by root-knot nematodes in white clover and is associated with the activation of the flavonoid pathway. Aust. J. Plant Physiol. 26:221-231.

Jones, M. G. K. 1981. The development and function of plant cells modified by endoparasitic nematodes. Pages 255-279 in: Plant Parasitic Nematodes, Vol. 3. B. M. Zukermann and R. A. Rohde, eds. Academic Press, New York.

Karczmarek, A., Overmars, H., Helder, J., and Goverse, A. 2004. Feeding cell development by cyst and root-knot nematodes involves a similar early, local and transient activation of a specific auxin-inducible promoter element. Mol. Plant Pathol. 5:343-346.

Karimi, M., Inzé, D., and Depicker, A. 2002. GATEWAY vectors for Agrobacterium-mediated plant transformation. Trends Plant Sci. S7:193-195.

Kudla, U., Milac, A. L., Qin, L., Overmars, H., Roze, E., Holterman, M., Petrescu, A. J., Goverse, A., Bakker, J., Helder, J., and Smant, G. 2007. Structural and functional characterization of a novel, host penetrationrelated pectate lyase from the potato cyst nematode Globodera rostochiensis. Mol. Plant Pathol. 8:293-305.

Lashbrook, C. C., Giovannoni, J. J., Hall, B. D., Fischer, R. L., and Bennett, A. B. 1998. Transgenic analysis of tomato endo- $\beta-1,4$-glucanase gene function. Role of cell in floral abscission. Plant J. 13:303-310.

Niebel, A., de Almeida Engler, J., Tiré, C., Engler, G., van Montagu, M., and Gheysen, G. 1993. Induction patterns of an extensin gene in tobacco upon nematode infection. Plant Cell 5:1697-1710.

Popeijus, H., Overmars, H., Jones, J., Blok, V., Goverse, A., Helder, J., Schots, A., Bakker, J., and Smant, G. 2000. Enzymology-degradation of plant cell walls by a nematode. Nature 406:36-37.

Qin, L., Kudla, U., Roze, E. H. A., Goverse, A., Popeijus, H., Nieuwland, J., Overmars, H., Jones, J. T., Schots, A., Smant, G., Bakker, J., and Helder, J. 2004. Plant degradation: A nematode expansin acting on plants. Nature 427:30.

Siebert, P. D., Chenchik, A., Kellogg, D. E., Lukyanov, K. A., and Lukyanov, S. A. 1995. An improved PCR method for walking in uncloned genomic DNA. Nucleic Acids Res. 23:1087-1088.

Smant, G., Stokkermans, J. P. W. G., Yan, Y., de Boer, J. M., Baum, T. J., Wang, X., Hussey, R. S., Gommers, F. J., Henrissat, B., Davis, E. L. Helder, J., Schots, A., and Bakker, J. 1998. Endogenous cellulases in animals: Isolation of $\beta-1,4$-endoglucanase genes from two species of plantparasitic cyst nematodes. Proc. Natl. Acad. Sci. U.S.A. 95:4906-4911.

Urbanowicz, B. R., Catalá, C., Irwin, D., Wilson, D. B., Ripoll, D. R., and Rose, J. K. C. 2007. A tomato endo- $\beta$-1,4-glucanase, SiCe19C1, represents a distinct subclass with a new family of carbohydrate binding modules (CBM49). J. Biol. Chem. 282:12066-12074.

van Engelen, F. A., Schouten, A., Molthoff, J. W., Roosien, J., Salinas, J., Dirkse, W. G., Schots, A., Bakker, J., Gommers, F. J., Jongsma, M. A., Bosch, D., and Stiekema, W. J. 1994. Coordinate expression of antibody subunit genes yields high levels of functional antibodies in roots of transgenic tobacco. Plant Mol. Biol. 26:1701-1710.

Vercauteren, I., de Almeida Engler, J., De Groodt, R., and Gheysen, G. 2002. An Arabidopsis thaliana pectin acetylesterase gene is upregulated in nematode feeding sites induced by root-knot and cyst nematodes. Mol. Plant-Microbe Interact. 15:404-407.

Wieczorek, K., Golecki, B., Gerdes, L., Heinen, P., Szakasits, D. Durachko, D. M., Cosgrove, D. J., Kreil, D. P., Puzio, P. S., Bohlmann, H., and Grundler, F. M. W. 2006. Expansins are involved in the formation of nematode-induced syncytia in roots of Arabidopsis thaliana. Plant J. 48:98-112. 